\title{
Uso da topografia de córnea na adaptação de lente de contato rígida gás-permeável em pacientes portadores de ceratocone: descrição de técnica e resultados preliminares
}

\author{
Corneal topography for rigid gas permeable lens fitting in keratoconus - technique and \\ preliminary results
}

\author{
José Álvaro Pereira Gomes ${ }^{1}$ \\ Luiz Alexandre Lani² \\ Yara Juliano ${ }^{3}$ \\ Roseli Gomes $^{2}$ \\ Erik Alessandro Pedro ${ }^{4}$ \\ Roberto Anbar 5
}

\begin{tabular}{|l|}
\hline \multicolumn{1}{|c|}{ RESUMO } \\
\hline Objetivo: Apresentar um método prático baseado nos valores topográfi- \\
cos para adaptação de lente de contato rígida gás-permeável (LCRGP) em \\
pacientes com ceratocone. Método: Foram estudados 33 olhos de 17 \\
pacientes consecutivos portadores de ceratocone, no período de julho de \\
1997 a abril de 1999. O exame inicial consistiu de medida da acuidade visual, \\
refração, biomicroscopia e topografia de córnea com o topógrafo compu- \\
tadorizado Eye Sys. As sessões de adaptação foram feitas com lentes de \\
contato de teste, com curva-base selecionada a partir da topografia de \\
córnea. Foi considerada como referência inicial o valor médio de K 1,5 mm \\
superior ao centro óptico de cada olho a $90^{\circ}$. Resultados: A adaptação de \\
lente de contato rígida gás-permeável foi bem sucedida em 30 olhos (91\%). \\
Em todos esses casos houve melhora significativa da acuidade visual, que \\
nofinal variou de $20 / 20$ a $20 / 60$. Para a adaptação inicial, foram necessárias, \\
em média, $3 \pm 1$ tentativas. Foi realizada readaptação com sucesso em 3 \\
casos (10\%). Na maioria dos casos (57\%), as lentes pedidas possuíam \\
curva-base com valor mais próximo de K 1,5 mm a $90^{\circ}$ em relação a K. Esse \\
achado foi comprovado por análise estatística das medidas individualmente \\
e das médias das diferenças entre as duas medidas topográficas e a curva- \\
base final de lente pedida. Conclusão: A adaptação baseada na topografia \\
de córnea $1,5 m m$ superiormente $90^{\circ}$ ao centro óptico mostrou-se rápida e \\
eficaz nos casos de ceratocone.
\end{tabular}

Descritores: Ceratocone; Lente de contato; Topografia da córnea; Astigmatismo; Adaptação ocular

\section{INTRODUÇÃ̃O}

Ceratocone é alteração ectásica da córnea, caracterizada pelo afinamento e protrusão central e/ou paracentral, que progressivamente assume a forma cônica. A doença inicia-se predominantemente na segunda década de vida e costuma progredir por cerca de 6 anos, estabilizando-se posteriormente. $\mathrm{O}$ afinamento progressivo, protrusão e cicatrização central estromal resultam em astigmatismo regular ou irregular, que causam diminuição da acuidade visual $^{(1)}$.

Estima-se que 74 a $87 \%$ dos pacientes com ceratocone obtenham correção satisfatória do erro refracional pelo emprego de recursos ópticos ${ }^{(2-3)}$. A correção com óculos nem sempre é possível, especialmente nos casos em que há astigmatismo irregular e/ou protrusão de grande magnitude ${ }^{(4)}$. Nesses casos, utilizam-se lentes de contato rígidas, de preferência gás-permeáveis, 
520 Uso da topografia de córnea na adaptação de lente de contato rígida gás-permeável em pacientes portadores de ceratocone: descrição de técnica e resultados preliminares

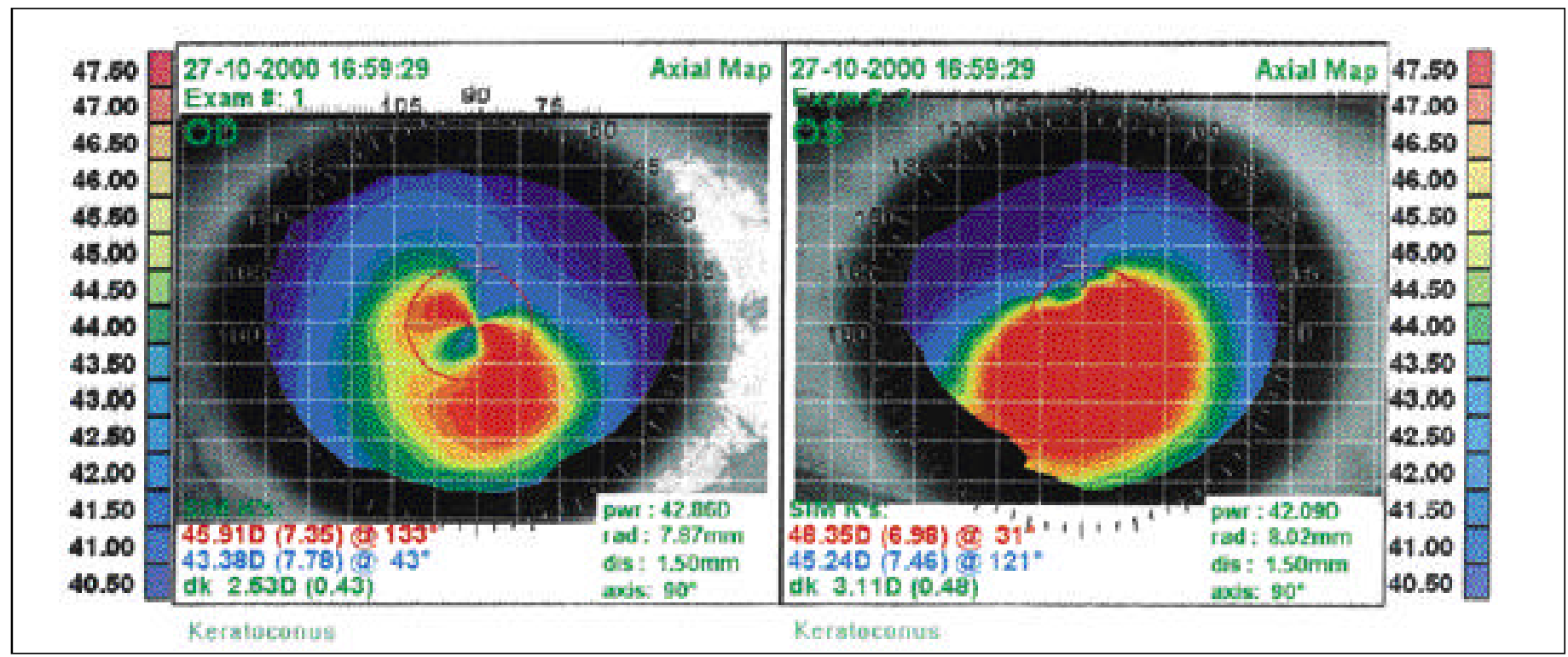

Figura 1 - Topografia da córnea de paciente com ceratocone, demonstrando o valor $1,5 \mathrm{~mm}$ superior ao eixo visual a $90^{\circ}$ (seta) para adaptação de lente contato RGP

que além de corrigir o astigmatismo irregular, proporcionando melhora da acuidade visual, possuem alta permeabilidade ao oxigênio $^{(4)}$. Nos casos que se apresenta impossibilidade de correção óptica com óculos ou lentes de contato, indica-se cirurgia, mais comumente ceratoplastia penetrante.

A ceratoscopia computadorizada proporcionou grande avanço no entendimento das alterações topográficas da córnea. É importante instrumento diagnóstico, especialmente do ceratocone, e pode ser utilizado na adaptação de lentes de contato $^{(4)}$.

O objetivo deste estudo é apresentar um método prático de adaptação de lente de contato rígida gás-permeável (LCRGP) baseado nos valores topográficos em pacientes com ceratocone.

\section{MÉTODO}

Foram estudados 33 olhos de 17 pacientes consecutivos portadores de ceratocone, cuja adaptação de óculos foi ineficaz para correção de sua ametropia, no período de julho de 1997 a abril de 1999. Dos 17 pacientes, 8 (47\%) eram do sexo masculino e 9 (53\%) do sexo feminino. A idade média dos pacientes foi de 25 anos, variando de 14 a 43 anos. Nove pacientes (53\%) referiam uso prévio de lentes de contato.

O exame inicial constou de medida da acuidade visual com e sem correção óptica, refração, biomicroscopia à lâmpada de fenda, mapeamento de retina e topografia de córnea com o sistema Eye Sys (Eye Sys system $2000^{\circledR}$, Eye Sys Technologies, Houston, EUA).

A adaptação de LCRGP foi feita com lentes de prova de acrílico ou gás-permeávis, de curva base selecionada a partir da topografia de córnea. Foi considerado como referência inicial o valor médio de K 1,5 mm superior ao centro óptico de cada olho a $90^{\circ(5)}$ (Figura 1). Não foi usado colírio anestésico durante as sessões de adaptação. Foi realizado exame à lâmpada de fenda para avaliar o padrão de coloração com fluoresceína imediatamente após a colocação da lente de teste. Nos casos em que a lente de teste se apresentava apertada, uma lente de menor diâmetro e/ou mais plana era testada. Quando a lente de teste se apresentava frouxa, uma lente de maior diâmetro e/ou mais apertada era testada. Quando a lente testada apresentava bom padrão de adaptação e centralização, e o paciente referia conforto, procedia-se a sobre-refração após 30 minutos para determinar o grau final da lente a ser solicitada ${ }^{(5)}$ (Figura 2). Assim que o paciente iniciava o uso da LCRGP, fazia controles mensalmente com exame oftalmológico completo. Considerou-se bem sucedida a adaptação da lente de contato que permitiu melhora da acuidade visual, conforto e tempo de uso maior do que 6 horas nos olhos com pelo menos dois meses de seguimento ${ }^{(6)}$.

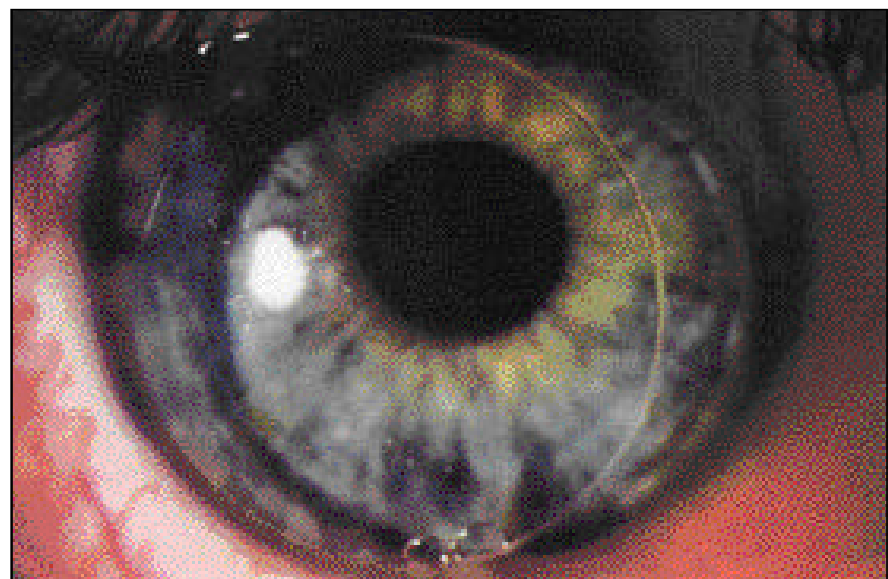

Figura 2 - Lente de contato RGP apresentando bom padrão de adaptação 


\section{Análise estatística}

A fim de comparar, para cada paciente, os valores da curva base final das lentes de contato pedidas com os valores de $\mathrm{K}$ (medida do meridiano mais plano da córnea) e K 1,5 mm superior ao centro óptico, foi realizada análise de variância para valores repetidos ${ }^{(7)}$. Foi também realizado teste de Wilcoxon com a finalidade de comparar as diferenças entre a curva base da lente pedida e as medidas de $\mathrm{K}$ e $\mathrm{K} 1,5 \mathrm{~mm}$ superior ao centro óptico ${ }^{(8)}$.

\section{RESULTADOS}

O tempo médio de seguimento foi de 11 meses (variação de 2 a 17 meses). A adaptação de LCRGP foi bem sucedida em 30 olhos (91\%). Em todos esses casos, houve melhora significativa da acuidade visual, que ao final variou de $20 / 20$ a 20/30 (87\%) e 20/30 a 20/60 (13\%). Para a adaptação inicial, foram necessárias, em média, $3 \pm 1$ tentativas. Foi realizada readaptação com sucesso em 3 casos (10\%). Os resultados encontramse expressos na tabela 2.

Em relação a análise topográfica qualitativa da córnea, observou-se que os ceratocones localizavam-se na região inferior $(85 \%)$, central $(6 \%)$ e superior $(9 \%)$ da córnea.

Em relação a análise topográfica quantitativa (Tabela 1), a curva base da lente de contato final possuía valor mais próximo de $\mathrm{K} 1,5 \mathrm{~mm}$ a $90^{\circ}$ em relação a $\mathrm{K}$ em $57 \%$ dos casos, enquanto que o contrário ocorreu em apenas 16\%. Nos 27\% dos casos restantes, os valores de $\mathrm{K} 1,5 \mathrm{~mm}$ a $90^{\circ}$ e de $\mathrm{K}$ apresentaram-se concordantes. Os valores médios calculados foram de 47,6 $\pm 5,0 \mathrm{D}$ para $\mathrm{Ke} 46,7 \pm 5,3 \mathrm{D}$ para $\mathrm{K} 1,5 \mathrm{~mm}$ superior ao centro óptico. A curva base final das lentes de contato pedidas foi de $45,3 \pm 3,4 \mathrm{D}$.

Ao examinarmos os valores individuais, a análise de variância mostrou diferença significante entre as curvas base das lentes pedidas e os valores de $\mathrm{K}$ e $\mathrm{K}$ 1,5 mm superior ao

\begin{tabular}{|c|c|c|c|c|c|c|c|}
\hline \multicolumn{8}{|c|}{ Tabela1. Dados dos pacientes com ceratocone e respectivas topografias } \\
\hline \multirow[b]{2}{*}{ Paciente } & \multirow[b]{2}{*}{ Olho } & \multirow[b]{2}{*}{ AV c/c } & \multicolumn{5}{|c|}{ Dados da topografia } \\
\hline & & & $\begin{array}{l}\text { Ceratometria } \\
\text { (Sim K) }\end{array}$ & $\begin{array}{c}\text { Curvatura } \\
\text { central média }\end{array}$ & $\begin{array}{c}\text { Valor de } \mathrm{K} 1,5 \mathrm{~mm} \\
\text { sup. a } 90^{\circ}\end{array}$ & $\begin{array}{l}\text { Curvatura média } \\
\text { do ápice do cone }\end{array}$ & $\begin{array}{l}\text { Localização } \\
\text { do cone }\end{array}$ \\
\hline 1 & OD & $20 / 60$ & $45,98 \times 97^{\circ} 41,51 \times 07^{\circ}$ & 45,06 & 41,67 & 52,02 & Central inferior \\
\hline 1 & OE & $20 / 40$ & $43,32 \times 82^{\circ} 40,37 \times 172^{\circ}$ & 41,34 & 39,40 & 48,84 & Central inferior \\
\hline 2 & OD & $20 / 25$ & $47,00 \times 123^{\circ} 44,70 \times 33^{\circ}$ & 46,78 & 43,49 & 51,65 & Temporal inferior \\
\hline 2 & $\mathrm{OE}$ & $20 / 25$ & $45,30 \times 60^{\circ} 43,66 \times 150^{\circ}$ & 44,70 & 43,08 & 47,91 & Central inferior \\
\hline 3 & OD & $20 / 40$ & $50,82 \times 136^{\circ} 47,40 \times 46^{\circ}$ & 50,06 & 42,13 & 59,12 & Central inferior \\
\hline 3 & OE & $20 / 60$ & $59,52 \times 160^{\circ} 56,53 \times 70^{\circ}$ & 68,17 & 48,74 & 74,13 & Central inferior \\
\hline 4 & OD & $20 / 40$ & $47,60 \times 145^{\circ} 47,20 \times 55^{\circ}$ & 48,35 & 44,54 & 51,41 & Nasal inferior \\
\hline 4 & OE & $20 / 50$ & $47,33 \times 63^{\circ} 46,61 \times 153^{\circ}$ & 48,95 & 44,81 & 50,62 & Central inferior \\
\hline 5 & OD & $20 / 30$ & $46,35 \times 147^{\circ} 42,50 \times 57^{\circ}$ & 43,00 & 40,38 & 48,66 & Central inferior \\
\hline 5 & OE & $20 / 30$ & $45,06 \times 25^{\circ} 42,82 \times 115^{\circ}$ & 42,74 & 41,00 & 48,62 & Central inferior \\
\hline 6 & OD & $20 / 70$ & $45,12 \times 82^{\circ} 43,83 \times 172^{\circ}$ & 44,16 & 45,16 & 45,25 & Central inferior \\
\hline 6 & $\mathrm{OE}$ & $20 / 100$ & $45,24 \times 83^{\circ} 43,60 \times 173^{\circ}$ & 43,90 & 45,31 & 45,37 & Central inferior \\
\hline 7 & OD & $20 / 200$ & $66,04 \times 92^{\circ}$ & 63,63 & 65,70 & 70,72 & Temporal Superior \\
\hline 7 & OE & $20 / 40$ & $44,58 \times 40^{\circ} 42,66 \times 130^{\circ}$ & 44,40 & 42,70 & 46,96 & Temporal superior \\
\hline 8 & OD & $20 / 100$ & $58,79 \times 132^{\circ} 52,73 \times 42^{\circ}$ & 60,00 & 51,15 & 62,00 & Nasal inferior \\
\hline 8 & $\mathrm{OE}$ & $20 / 40$ & $52,73 \times 42^{\circ}$ & 55,00 & 46,75 & 61,00 & Central inferior \\
\hline 9 & OD & $20 / 100$ & $60,26 \times 115^{\circ} 55,87 \times 25^{\circ}$ & 56,00 & 52,96 & 59,00 & Central inferior \\
\hline 9 & OE & $20 / 80$ & $51,44 \times 54^{\circ} 50,14 \times 44^{\circ}$ & 53,00 & 49,48 & 56,00 & Central inferior \\
\hline 10 & OD & $20 / 100$ & $58,59 \times 134^{\circ} 56,46 \times 43^{\circ}$ & 62,05 & 51,25 & 57,23 & Nasal inferior \\
\hline 11 & OD & $20 / 200$ & $63,43 \times 161^{\circ} 57,79 \times 90^{\circ}$ & 58,82 & 62,50 & 62,50 & Central superior \\
\hline 11 & $\mathrm{OE}$ & $20 / 60$ & $50,59 \times 161^{\circ} 45,58 \times 90^{\circ}$ & 53,18 & 45,00 & 45,00 & Central inferior \\
\hline 12 & OD & $20 / 200$ & $54,08 \times 135^{\circ} 50,67 \times 45^{\circ}$ & 53,20 & 55,50 & 49,43 & Nasal inferior \\
\hline 12 & OE & $20 / 40$ & $49,70 \times 32^{\circ} 47,13 \times 122^{\circ}$ & 49,59 & 53,00 & 44,83 & Nasal inferior \\
\hline 13 & OD & $20 / 30$ & $46,10 \times 130^{\circ} 45,06 \times 40^{\circ}$ & 42,75 & 41,88 & 41,08 & Temporal inferior \\
\hline 13 & OE & $20 / 40$ & $50,14 \times 24^{\circ} 49,98 \times 114^{\circ}$ & 42,50 & 46,75 & 40,75 & Temporal inferior \\
\hline 14 & OD & $20 / 80$ & $48,35 \times 70^{\circ} 47,00 \times 160^{\circ}$ & 49,23 & 46,89 & 51,00 & Cemtral inferior \\
\hline 14 & OE & $20 / 40$ & $44,88 \times 180^{\circ} 44,40 \times 90^{\circ}$ & 45,15 & 44,17 & 46,60 & Central \\
\hline 15 & OD & $20 / 80$ & $47,80 \times 114^{\circ} 44,58 \times 24^{\circ}$ & 46,10 & 44,25 & 49,50 & Central temporal \\
\hline 15 & $\mathrm{OE}$ & CD a $1 \mathrm{~m}$ & $59,10 \times 44^{\circ} 53,74 \times 134^{\circ}$ & 62,83 & 49,50 & 49,50 & Central inferior \\
\hline 16 & OD & $20 / 200$ & $49,70 \times 141^{\circ} 46,04 \times 51^{\circ}$ & 50,68 & 42,00 & 46,50 & Central inferior \\
\hline 16 & OE & $20 / 40$ & $43,32 \times 58^{\circ} 43,15 \times 148^{\circ}$ & 42,84 & 43,00 & 44,00 & Central inferior \\
\hline 17 & OD & $20 / 200$ & $55,30 \times 60^{\circ} 53,06 \times 166^{\circ}$ & 56,65 & 56,48 & 57,65 & Central inferior \\
\hline 17 & OE & $20 / 200$ & $59,52 \times 64^{\circ} 52,73 \times 154^{\circ}$ & 58,60 & 50,84 & 50,84 & Central inferior \\
\hline
\end{tabular}


522 Uso da topografia de córnea na adaptação de lente de contato rígida gás-permeável em pacientes portadores de ceratocone: descrição de técnica e resultados preliminares

\begin{tabular}{|c|c|c|c|c|c|c|c|c|}
\hline \multirow[b]{2}{*}{ Paciente } & \multirow[b]{2}{*}{ Olho } & & & & LC fir & & & \\
\hline & & Tentativas & Readaptação & Grau final LC & Curvatura & Diâmetro & $A V \mathrm{c} / \mathrm{LC}$ & Seguimento \\
\hline 1 & OD & 1 & 0 & $-2,00$ & 42,00 & 8,8 & $20 / 25$ & $1 \mathrm{a} 4 \mathrm{~m}$ \\
\hline 1 & $\mathrm{OE}$ & 2 & 0 & $-1,25$ & 41,00 & 8,9 & $20 / 25$ & \\
\hline 2 & OD & 3 & 0 & $-3,50$ & 43,00 & 9,6 & $20 / 20$ & 1 a $3 m$ \\
\hline 2 & $\mathrm{OE}$ & 3 & 0 & $-3,50$ & 42,00 & 9,6 & $20 / 20$ & 1 a $3 m$ \\
\hline 3 & OD & 4 & 1 & . $+0,50$ & 44,50 & 8,6 & $20 / 30$ & 1 a $4 m$ \\
\hline 3 & $\mathrm{OE}$ & 5 & 0 & $-3,75$ & 48,00 & 8,5 & $20 / 30$ & 1 a $4 m$ \\
\hline 4 & OD & 2 & 1 & $-4,00$ & 44,00 & 8,9 & $20 / 20$ & 1 a $2 m$ \\
\hline 4 & $\mathrm{OE}$ & 2 & 1 & $-5,25$ & 44,00 & 8,9 & $20 / 20$ & 1 a $2 m$ \\
\hline 5 & $O D$ & 1 & 0 & $-2,75$ & 40,50 & 8,9 & $20 / 20$ & 1 a $2 m$ \\
\hline 5 & $\mathrm{OE}$ & 1 & 0 & $-2,75$ & 40,25 & 8,9 & $20 / 20$ & 1 a $2 m$ \\
\hline 6 & OD & 3 & 0 & $-2,50$ & 43,50 & 9,6 & $20 / 20$ & 1 a $5 m$ \\
\hline 6 & $\mathrm{OE}$ & 2 & 0 & $-2,50$ & 43,50 & 9,6 & $20 / 20$ & 1 a $5 m$ \\
\hline 7 & OD & 3 & 0 & & & & & \\
\hline 7 & $\mathrm{OE}$ & 3 & 0 & $-3,00$ & 41,75 & 9,6 & $20 / 25$ & 1 a $2 m$ \\
\hline 8 & OD & 5 & 0 & $-5,00$ & 49,50 & 8,7 & $20 / 50$ & $11 \mathrm{~m}$ \\
\hline 8 & $\mathrm{OE}$ & 5 & 0 & $-4,00$ & 48,75 & 9,0 & $20 / 30$ & $11 \mathrm{~m}$ \\
\hline 9 & $O D$ & 2 & 0 & $-6,00$ & 49,50 & 8,4 & $20 / 30$ & 1 a $2 m$ \\
\hline 9 & $\mathrm{OE}$ & 1 & 0 & $-5,00$ & 49,00 & 8,7 & $20 / 20$ & 1 a $2 m$ \\
\hline 10 & OD & 2 & 0 & $-5,00$ & 49,00 & 8,5 & $20 / 20$ & $11 \mathrm{~m}$ \\
\hline 11 & OD & 5 & 0 & $-9,50$ & 52,00 & 8,5 & $20 / 40$ & 1 a $5 m$ \\
\hline 11 & $\mathrm{OE}$ & 4 & 0 & $-4,00$ & 46,50 & 8,6 & $20 / 25$ & 1 a $5 \mathrm{~m}$ \\
\hline 12 & OD & 2 & 0 & $-7,50$ & 49,00 & 8,7 & $20 / 30$ & $6 \mathrm{~m}$ \\
\hline 12 & $\mathrm{OE}$ & 1 & 0 & $-2,00$ & 44,75 & 8,7 & 20/20 & $6 m$ \\
\hline 13 & $O D$ & 3 & 0 & $-1,50$ & 42,75 & 9,6 & $20 / 25$ & $4 \mathrm{~m}$ \\
\hline 13 & $\mathrm{OE}$ & 3 & 0 & $-1,00$ & 42,50 & 9,6 & 20/20 & $4 \mathrm{~m}$ \\
\hline 14 & OD & 5 & 0 & $-2,00$ & 44,50 & 8,6 & $20 / 30$ & $3 \mathrm{~m}$ \\
\hline 14 & $\mathrm{OE}$ & 1 & 0 & $-2,00$ & 44,25 & 8,8 & $20 / 30$ & $3 m$ \\
\hline 15 & $O D$ & 4 & 0 & $-0,50$ & 44,00 & 8,6 & $20 / 30$ & $2 m$ \\
\hline 15 & $\mathrm{OE}$ & 3 & 0 & $-5,75$ & 47,50 & 8,5 & $20 / 60$ & $2 m$ \\
\hline 16 & $O D$ & 4 & 0 & $-4,00$ & 43,50 & 9,6 & $20 / 40$ & $6 \mathrm{~m}$ \\
\hline 16 & $\mathrm{OE}$ & 3 & 0 & $-4,50$ & 42,50 & 9,6 & $20 / 25$ & $6 m$ \\
\hline 17 & OD & 5 & 0 & $-5,75$ & 52,00 & 8,6 & $20 / 30$ & $10 \mathrm{~m}$ \\
\hline 17 & $\mathrm{OE}$ & 4 & 0 & $-5,50$ & 49,00 & 8,5 & $20 / 25$ & $10 \mathrm{~m}$ \\
\hline
\end{tabular}

centro óptico $[\mathrm{F}$ calculado $=9,68(\mathrm{p}<0,001)]$. Observou-se que as diferenças tenderam a ser maiores em relação a $K$.

Para comparar as diferenças entre curva base das lentes pedidas e os valores de $\mathrm{K}$ e K 1,5 mm superior ao centro óptico, foram calculadas suas médias, que foram: Curvatura da LC-K $=-2,33$; Curvatura da LC $-(\mathrm{K} 1,5 \mathrm{~mm})=-1,47$. Ao compararmos esses valores pelo teste de Wilcoxon, encontramos z calculado $=1,91(\mathrm{p}=0,0281)$, que mostrou que as diferenças entre a lente de contato pedida e $\mathrm{K} 1,5 \mathrm{~mm}$ superior ao centro óptico foram significantemente menores do que as calculadas em relação aos valores de $\mathrm{K}$.

\section{DISCUSSÃO}

No ceratocone, nota-se que a área apical da área ectásica encontra-se deslocada do centro visual da córnea ${ }^{(4,9)}$. Essa área apical costuma ser, nesses casos, significantemente mais curva do que a área do centro visual, o que dificulta a adaptação das lentes de contato rígidas com apenas uma curvatura posterior ${ }^{(9)}$.
Diferentes tipos e técnicas de adaptação de lentes de contato têm sido utilizados para a correção do erro refracional dos pacientes portadores de ceratocone. Em 1969, Soper introduziu uma lente de contato que possui curvatura posterior central constituída de duas zonas - uma zona central com curvatura adaptada na região apical do cone; e uma zona periférica de curvatura constante de $45 \mathrm{D}$ ou $7,5 \mathrm{~mm}^{(9)}$. Outros tipos de lentes, como a Ni-cone, com três zonas posteriores de curvatura posterior; lente asférica de Mc Guire; e a lente híbrida Softperm, com área central rígida e perférica gelatinosa, constituem opções nos casos de ceratocone ${ }^{(9)}$. O "piggyback" pode ser definido como a adaptação de uma lente de contato gelatinosa, sobre a qual se adapta uma lente de contato rígida ${ }^{(9)}$. Esse tipo de adaptação tem sido empregado com sucesso em pacientes com problemas de adaptação de lentes de contato rígida.

Apesar das diferentes opções disponíveis, a primeira opção continua sendo a lente de contato rígida gás-permeável ${ }^{(9-10)}$. O baixo custo, a facilidade de encontrar lentes para teste, a obtenção de boa acuidade visual e a pronta entrega são al- 
guns dos argumentos a favor de, quando possível, tentar adaptar esse tipo de lente de contato nos pacientes com ceratocone $^{(10)}$.

Muitos oftalmologistas costumam tentar adaptar LCRGP baseados no $\mathrm{K}$ médio, procurando posicionar a lente no ápice do cone ${ }^{(9)}$. A justificativa é a de tentar corrigir a área irregular proporcionada pelo cone para obter boa acuidade visual. Outros autores preferem adaptar a LCRGP no K mais plano (ou K), procurando posicionar a lente numa posição mais superior e de forma menos $\operatorname{apertada}^{(5,11)}$.

A adaptação da LCRGP baseada no valor médio de K 1,5 mm superior a $90^{\circ}$ foi primeiramente descrita por Lopatynsky et al., em 1993, para corrigir astigmatismo pós-transplante de córnea $^{(5)}$. O intuito desse tipo de adaptação é o de promover uma adaptação superior e mais plana, e por isso o valor do $\mathrm{K}$ da lente deve se basear no valor da curvatura da córnea sobre a qual a lente deve ficar, ou seja, superior. Num trabalho subseqüente, Gomes et al. constataram não haver alterações corneanas topográficas e clínicas significativas em pacientes pós-transplante de córnea que estavam utilizando LCRGP adaptadas, conforme a técnica descrita por Lopatynsky, após período de $3 \operatorname{anos}^{(12)}$. Muitos desses pacientes apresentavam ceratometria central média com valores superiores a $46 \mathrm{D}^{(5,12)}$.

Neste trabalho, propusemo-nos a adaptar LCRGP em pacientes portadores de ceratocone utilizando o mesmo tipo de adaptação proposto por Lopatynsky. Apesar do grau de ceratocone apresentado pelos pacientes, $36 \%$ com ceratocone de grau avançado e severo (acima de 52D), obtivémos uma taxa de sucesso de $91 \%$, após um número relativamente pequeno de tentativas (média de 3 tentativas por lente). A adaptação superior proporcionou boa visão (87\% com AV melhor que 20/30) e conforto nesses pacientes, muitos dos quais portadores de atopia e com história pregressa de dificuldade de adaptação de lentes de contato.

É importante mencionar que, na maioria dos casos, a lente de contato final possuía curva base com valor mais próximo do $\mathrm{K} 1,5 \mathrm{~mm}$ a $90^{\circ}$ do que de $\mathrm{K}$. Esse resultado foi comprovado por análise estatística das medidas individualmente e das médias das diferenças entre as duas medidas topográficas e a curva base final de lente pedida. Cabe ressaltar que, mesmo levandose em consideração que o teste inicial foi baseado no valor de $\mathrm{K} 1,5 \mathrm{~mm}$ a $90^{\circ}$, poderíamos ter encontrado curva base da lente de contato final diferente desse valor e mais próxima de K. No entanto, o resultado por nós encontrado reforça a tese de que a adaptação baseada no K 1,5 mm superior a $90^{\circ}$ é mais adequada para o posicionamento superior de LCRGP nos casos de astigmatismos acentuados e/ou irregulares, como os encontrados após transplante de córnea e ceratocone. Além disso, é fácil de ser determinada pela topografia de córnea, dispensando a necessidade de "softwares" mais complexos, dispendiosos e de discutível valor prático.

\section{CONCLUSÃO}

A adaptação de LCRGP baseada no valor médio de K 1,5 mm superior a $90^{\circ}$ obtido pela topografia de córnea mostrou-se rápida e eficaz nos casos de ceratocone. Trabalhos controlados, comparativos prospectivos e com maior número de pacientes são necessários para comprovar esses achados.

\section{ABSTRACT}

Purpose: To introduce a practical method based on the topographic values for rigid gas-permeable contact lens (RGPCL) fitting in keratoconus patients. Methods: Thirty-three eyes of 17 consecutive patients with keratoconus were studied between July 1997 and April 1999. The initial ophthalmologic examination consisted of visual acuity examination, refraction, biomicroscopy, corneal topography with Eye Sys system. The tested contact lenses were selected based on results of corneal topography $1.5 \mathrm{~mm}$ superiorly from the visual axis at $90^{\circ}$ the base curve. Results: Rigid gas-permeable contact lens fitting was successful in 30 eyes $(91 \%)$. There was improvement in visual acuity in all eyes, ranging from $20 / 20$ to $20 / 60$. A mean of $3 \pm 1$ tests were performed to select the final contact lenses parameters. Refitting was necessary in 3 cases $(10 \%)$. The majority of the rigid gas-permeable contact lenses (57\%) had their base curves similar to $\mathrm{K} 1.5 \mathrm{~mm}$ superiorly from the visual axis compared to $\mathrm{K}$ value. This finding was confirmed with statistical analysis of the individual measurements and the mean differences between the two topographic measurements and the final base curve of the lens. Conclusions: Rigid gas-permeable contact lens fitting based on the corneal topography $1.5 \mathrm{~mm}$ superior from the visual axis at $90^{\circ}$ showed to be easy and efficient for eyes with keratoconus.

Keywords: Keratoconus; Contact lenses; Corneal topography; Astigmatism; Ocular adaptation

\section{REFERÊNCIAS}

1. Bechara SJ, Kara-José N. Ceratocone. In: Belfort Jr R, Kara-José N editores. Córnea Clínica-Cirúrgica. São Paulo: Editora Roca Ltda; 1997. p.359-66.

2. Lass JH, Lembach RG, Park SB. Clinical management of keratoconus. Ophthalmology 1990;97:433-45.

3. Johnson DA, O`Brien T, Stark WJ. Penetrating keratoplasty for keratoconus? (letter). J Cataract Refract Surg 1997;23:1130-1.

4. Buxton JN, Buxton DF, Dias AK, Scorsetti DH. Keratoconus basic clinic and features. Kastl PR editor. Contact Lenses: The CLAO Guide to Basic Science and Clinical Practice. Dubuque: Kendall/Hunt Publishing Company; 1995. p.101-21.

5. Lopatynsky M, Cohen EJ, Leavitt KG, Labison PR. Corneal Topography for Rigid Gas Permeable Lens Fitting After Penetrating Keratoplasty. CLAO, 1993;19:41-4.

6. Pullum KW, Buckley RJ. A study of 530 patients referred for rigid gas permeable scleral contact lens assessment. Cornea 1997;16:612-622.

7. Sokal RR, Rohlf FJ. Biometry. São Francisco: WH Freeman \& Company; 1969.

8. Siegel S, Castellan Jr. NJ. Non-parametric statistics (2 ${ }^{\text {a }}$ Edição). Nova Iorque: McGraw, Hill Int. Ed.; 1988.

9. Soper JW, Soper MP. Keratoconus contact lens fitting. Kastl RP editor. Contact Lenses: The CLAO Guide to Basic Science and Clinical Practice. Dubuque: Kendall/Hunt Publishing Company; 1995. p.123-9.

10. Key J, Benett SE. Rigid Gas-Permeable (RGP) Extended Wear Contact Lenses Kastl RP ed. Contact Lenses: The CLAO Guide to Basic Science and Clinical Practice. Dubuque: Kendall/Hunt Publishing Company; 1995. p.51-74.

11. Wasserman D, Itzkowitz J, Kamenar T, Asbell PA. Corneal topographic data: Its use in fitting aspheric contact lenses. CLAO 1992;18:83-5.

12. Gomes JAP, Rapuano CJ, Cohen EJ. Topographic Stability and Safety of Contact Lens Use After Penetrating Keratoplasty. CLAO 1996;22:64-9. 